\section{RETROSPECTIVE EXPOSURE ASSESSMENT ON THE PHOTOLITHOGRAPHY PROCESS IN SEMICONDUCTOR MANUFACTURING FACILITY}

${ }^{1}$ Seok Won Lee, ${ }^{1}$ Young Hee Yoon, ${ }^{1}$ Kyoung Ho Lee, ${ }^{1}$ Hyun Hee Jung, ${ }^{2}$ Kwan Sick Kim, 1,30ng II Kim. 'Samsung Health Research Institute, Samsung Electronics Co., Ltd., Yongin, Republic of Korea; 'Environment Safety Center, Samsung Electronics Co., Ltd., Yongin, Republic of Korea; ${ }^{3}$ Kangbuk Samsung Hospital, School of Medicine, Sungkyunkwan University, Seoul, Republic of Korea

10.1136/oemed-2014-102362.217

Objectives The purpose of this study was to assess potential occupational exposure to chemicals among workers using both qualitative and quantitative information during specific job tasks on the photolithography process of a semiconductor manufacturing facility.

Method Historical exposure measurement data from 1997 to 2012 ( $\mathrm{n}=1347$ ) were collected and classified by each type of chemical during 8 of 36 job tasks, and hallmark risk assessment was performed to determine control bands by job task of interest using the qualitative information on health hazard, duration of use, quantity, exposure control, etc. Finally, Bayesian Decision Analysis was performed to evaluate potential exposure ratings for those 8 job tasks by combining the prior distribution and likelihood distribution into the posterior distribution.

Results Control bands for 8 job tasks of interest were determined as 1 , interpreting that workers use appropriate industrial hygiene, follow recommended job practices, wear personal protective equipment, and instal proper ventilation systems. Arithmetic mean and standard deviation for 7 types of chemicals from historical exposure measurement data were 2-heptanone $0.013 \pm 0.011 \mathrm{ppm}, 1$-methoxypropyl-2-acetate $0.163 \pm 0.261$ ppm, acetone $0.456 \pm 0.609 \mathrm{ppm}$, ethyl 3-ethoxy propionate $0.131 \pm 0.106 \mathrm{ppm}$, ethyl lactate $0.058 \pm 0.067 \mathrm{ppm}$, isopropyl alcohol $0.594 \pm 0.923 \mathrm{ppm}$, and n-butyl acetate $0.071 \pm 0.368$ ppm, respectively, and all concentrations of chemicals were below $10 \%$ of OELs. Therefore, exposure ratings of the 8 job tasks were determined as exposure category 1, indicating that the 95 th percentile of exposures infrequently exceed $10 \%$ of the OELs.

Conclusions In conclusion, the study findings suggest that potential exposures to chemicals of interest among semiconductor workers for 16 years were very low (below 10\% of the OELs) and "highly controlled."

\section{THE ASSOCIATION BETWEEN STATINS USE AND RISK OF HOSPITALIZATIONFOR ACUTE HEPATITIS IN PATIENTS WITH HBV INFECTION}

${ }^{1}$ Pau-Chung Chen, ${ }^{1}$ Meng-yin Chen, ${ }^{1}$ Yu-Tse Tsan, ${ }^{1}$ Yao-Hsu Yang, ${ }^{2}$ Meng-Hung Lin. ${ }^{1}$ Institute of Occupational Medicine and Industrial Hygiene, Taipei, Taiwan; ${ }^{2}$ School of Medicine, China Medical University, Taichung, Taiwan, Taichung, Taiwan

\subsection{6/oemed-2014-102362.218}

Objectives Statins are commonly used to prevent coronary heart disease and stroke, but they may increase the risk of hepatotoxicity and muscle toxicity. Hepatitis B virus (HBV) infected patients are more susceptible to acute hepatitis. However, the safety of statins use in patients with HBV infection is unclear. The purpose of this study was to investigate the association between statins use and risk of hospitalizations related to acute hepatitis in patients with $\mathrm{HBV}$ infection.
Method Ours study was a population-based retrospective cohort study. We used a nationwide data of HBV-infected patient from 1999 to 2010 from the Taiwanese National Health Insurance database. Cox proportional hazards regression was used to calculate the hazard ratios (HRs) and 95\% CIs for the association between statins use and the occurrence of acute hepatitis in the HBV-infected study cohort.

Results A total of $255344 \mathrm{HBV}$-infected patients were included in the study cohort. Of these patients, 127,672 (50\%) had used statins, and $283(0.11 \%)$ were diagnosed with acute hepatitis within one year and incident rate of acute hepatitis in patients with HBV infection was 110.9 per 100000 person-years. After controlling for potential confounders, no association of acute hepatitis between statins user and non-statin user was found in different follow-up periods and doses within one year.

Conclusions Among patients with HBV infection, statins use may be not association with acute hepatitis regardless of larger cumulative dose, drug class of statins. Statins given to HBVinfection patients may not associate with severe liver injury.

\section{DIABETES MANAGEMENT AT WORK}

Kandouci Chahrazed, Belhadj Zoubida, Arab Aicha, Badreddine Abdelkrim Kandouci. University of Sidi Bel Abbes, Sidi Bel Abbes, Algeria

\subsection{6/oemed-2014-102362.219}

Objectives This study aims to demonstrate the interaction between diabetes and work, but also to identify any difficulties that a diabetic patient can meet at his place of work, which could hinder the daily management of the disease.

Method This is a descriptive, cross-sectional study that was conducted in the month of September 2012 until April 2013. The support for the survey is a pre anonymous self-administered questionnaire, among diabetic patients in employment in various institutions under agreement with the Department of Occupational Medicine of the University Hospital of Sidi Bel-Abbes during their periodic visits.

Results

- The final sample consisted of 83 diabetics with $61.4 \%$ men and $38.6 \%$ women, the average age of employees is 50 years, 62 . $6 \%$ of our patients are non-insulin-treated, $77.1 \%$ are type 2 diabetes, $68.7 \%$ for patients work schedules are compatible with meals and taken the treatment, and $65.1 \%$ find it difficult to follow the diet to work, 53\% reported hypoglycemia at work with variable severity.

- The type of diabetes is strongly linked to hypoglycemia occurred in the workplace and change of occupation $(\mathrm{p}=$ $0.022, \mathrm{p}=0.008)$

- Two factors related to the disease: duration of illness affects the occurrence of hypoglycemia $(p=0.014)$ and has a link with the achievement of capillary blood glucose and Modified Work.

Conclusions We have seen with the study, some socio-professional factors and factors related to the disease can occur in diabetes management.

\section{OCCUPATIONAL INJURIES AT THE COMMUNITY LEVEL}

Linda Forst, Brian Chin, Lee Friedman. University of Illinois at Chicago, Chicago, Illinois, USA

10.1136/oemed-2014-102362.220 